\title{
Effectiveness and Predictors of Poor Prognosis Following Intravenous Thrombolysis in Patients with Wake-Up Ischemic Stroke Guided by Rapid MRI
}

\author{
Qiuyun Lu $\mathbb{D}^{1, *}$, Qingke Bai ${ }^{2, *}$, Haiyan Ren', Benju Zhu', Tianfang Jiang', Chen Peng', Xu Chen' \\ 'Department of Neurology, Shanghai Eighth People's Hospital, Shanghai, People's Republic of China; ${ }^{2}$ Department of Neurology, Pudong New Area \\ People's Hospital, Shanghai, People's Republic of China \\ *These authors contributed equally to this work
}

Correspondence: Xu Chen, Department of Neurology, Shanghai Eighth People's Hospital, No. 8 Caobao Road, Shanghai, 200235, People's Republic of China, Tel +8619802103956, Fax+86021645I4326, Email cxwp65@I63.com

\begin{abstract}
Purpose: Our aim was to investigate the effectiveness and predictors of poor prognosis in WUIS patients who received alteplase thrombolysis under the guidance of diffusion-weighted imaging (DWI)-T2-weighted imaging (T2WI) mismatch.

Patients and Methods: We recruited patients within $4.5 \mathrm{~h}$ of acute ischemic stroke (AIS) and WUIS patients with uncertain onset times from two stroke centers. To evaluate effectiveness, we compared National Institutes of Health Stroke Scale (NIHSS) scores between the two groups at admission and at 24 h, 3 days, and 1 week thereafter. We also compared the two groups with respect to the modified Rankin Scale (mRS) score at 90 days after thrombolysis. The WUIS patients were divided into a favorable prognosis group ( $\mathrm{mRS}$ score: $0-1$ points) and a poor prognosis group (mRS score $\geq 2$ points). Data were compared between the two subgroups to identify factors that influence poor prognoses. The receiver operating characteristic (ROC) curve was used to evaluate the predictive value of factors related to poor prognosis.

Results: A total of 114 patients with WUIS and 316 patients with AIS were enrolled in the study. There were no significant differences between the two groups in terms of NIHSS or 90-day mRS scores $(p>0.05)$. Baseline NIHSS score (odds ratio [OR] $=$ $1.490,95 \%$ confidence interval $[\mathrm{CI}] 1.248-1.779, p<0.001)$ and atrial fibrillation (OR $=3.825,95 \% \mathrm{CI} 1.218-12.016, p=0.022)$ were identified as independent predictors of poor prognosis following thrombolysis in WUIS patients. The combined ROC diagnosis of these two variables had an area under the curve of 0.850 .
\end{abstract}

Conclusion: The DWI-T2WI sequence is an effective method to guide intravenous thrombolytic therapy for WUIS. Baseline NIHSS score and atrial fibrillation were identified as independent predictors of poor prognosis following thrombolysis in WUIS patients.

Keywords: rapid MRI, wake-up stroke, intravenous thrombolysis, effectiveness, poor prognosis

\section{Introduction}

Wake-up ischemic stroke (WUIS) refers to a form of acute cerebral infarction in which the patient falls asleep completely normally or has no new stroke symptoms. After awakening, patients exhibit certain stroke symptoms, such as neurological deficits. ${ }^{1}$ Patients with this condition are often excluded from receiving reperfusion treatment based on a specific time window because the exact onset time cannot be established. Thus, some patients are deemed unsuitable for thrombolysis and fail to receive timely treatment, which has serious consequences for clinical prognosis. ${ }^{2}$ Fortunately, the WAKE-UP study, published in 2018, described the use of the diffusion-weighted imaging (DWI)-fluid-attenuated inversion recovery (FLAIR) mismatch method to evaluate patients with acute ischemic stroke (AIS) who awaken more than $4.5 \mathrm{~h}$ from the last normal time or who have an unknown onset time. Compared with placebo, intravenous thrombolytic treatment significantly increases the proportion of patients who achieve good outcomes. ${ }^{3}$ Owing to the development of imaging technology, WUIS patients who are suitable for vascular recanalization therapy can now be 
identified; thus, the time constraint factor can be eliminated and the appropriate steps for clinical intervention can be taken. $^{4-6}$

A previous retrospective cohort study described the significant impact of every additional 1-minute delay in door-toneedle time (DNT); for each 1-minute delay, there was a $0.6 \%$ reduction in the odds of survival, a $0.3 \%$ increase in the odds of intracerebral hemorrhage, a $0.3 \%$ reduction in the activities of daily living, and a $0.4 \%$ reduction in living conditions and mobility. ${ }^{7}$ Another study calculated the lifetime benefits for every 1-min reduction in the onset-to-needle time (ONT) and found that, on average, each minute saved 1.8 days of healthy life. ${ }^{8}$ These findings demonstrate that every second counts when attempting to restore ischemic penumbra. Thus, it is essential that delays in hospital are reduced, particularly for patients with WUIS, in whom the onset time is uncertain. The imaging time of the T2-weighted imaging (T2WI) sequence is shorter than that of FLAIR because the former does not require compression of the cerebrospinal fluid signal, which can further shorten the DNT before alteplase thrombolysis and possibly restore more ischemic penumbra. However, imaging studies on WUIS have mostly focused on the DWI-FLAIR sequence, and few studies have investigated the DWI-T2WI sequence for guiding intravenous thrombolytic therapy for WUIS. Therefore, in the present study, we investigated the effectiveness and predictors of poor prognosis in WUIS patients who receive alteplase thrombolysis under the guidance of DWI-T2WI mismatch, given that the curative effects and prognoses of WUIS patients associated with this methodology remain the focus of much attention.

\section{Materials and Methods}

\section{Patient Selection}

We reviewed the clinical data of patients receiving intravenous thrombolysis with alteplase at Shanghai Eighth People's Hospital and Pudong New Area People's Hospital between July 2016 and June 2021. The inclusion criteria for patients in the WUIS group were as follows: aged 18-85 years; patients with neurological deficits caused by AIS and a National Institutes of Health Stroke Scale (NIHSS) score $\geq 4$ points; DWI-T2WI mismatch (ie, high DWI signal and negative T2WI); no intracranial hemorrhage (ICH) or obvious hypodensity changes of infarction on computed tomography (CT); no other contraindications for thrombolysis, as indicated by alteplase; and the patient or a family member provided written informed consent.

The inclusion criteria for patients in the control group were as follows: aged 18-85 years, patients with neurological deficits caused by AIS and an NIHSS score $\geq 4$ points; time of onset of less than $4.5 \mathrm{~h}$; high DWI signal and no obvious high T2WI signal; no ICH or obvious hypodensity changes of infarction on CT; no other contraindications for thrombolysis, as indicated by alteplase; and the patient or family member provided written informed consent.

\section{Data Collection}

From each patient, we collected various information, which included sex, age, medical history and/or newly diagnosed diseases after admission (eg, hypertension, hyperlipidemia, diabetes, atrial fibrillation), smoking history, systolic/diastolic blood pressure on admission, Trial of Org 10172 in Acute Stroke Treatment (TOAST) classification (ie, large artery atherosclerosis, small vessel occlusion, cardioembolism, other determined etiology, or undetermined etiology), NIHSS score, lesion site (ie, anterior or posterior circulation), laboratory examination (ie, glycosylated hemoglobin, fasting blood glucose, cholesterol, triglyceride, low-density lipoprotein cholesterol, homocysteine, and serum uric acid).

\section{Imaging Examinations and Treatment}

All patients underwent a brain CT scan to exclude ICH and early hypodensity signs. Patients with AIS were immediately examined using T2WI, DWI, and magnetic resonance angiography (MRA) sequences on a GE 1.5 Tesla HDXT Twinspeed magnetic resonance imaging (MRI) scanner. Intravenous thrombolytic drugs were prepared while informed consent was obtained. Images were analyzed and a consensus was reached by a radiologist and a neurologist. For intravenous thrombolytic therapy, patients in both groups received intravenous alteplase at a dose of $0.9 \mathrm{mg} / \mathrm{kg} ; 10 \%$ was injected intravenously within $1 \mathrm{~min}$, and the remaining 90\% was intravenously administered within 60 min (total dose was no more than $90 \mathrm{mg}$ ). Vital signs and neurological deficit scores were closely monitored. Brain CT was re-examined 
at any time if any changes were noted, which included aggravation of the original condition or the appearance of new neurological deficit symptoms (eg, deficits in language, cognition, motor function, gaze, and consciousness, visual field impairment, or visual neglect). Patients with WUIS were monitored using long-term dynamic electrocardiography (ECG) for 2 weeks.

\section{Effectiveness and Prognosis}

To evaluate effectiveness, we compared NIHSS scores between the two groups of patients at admission, and then at 24 hours, 3 days, and 1 week thereafter. We also compared between the two groups, the modified Rankin Scales (mRS) score at 90 days after thrombolysis. We recorded the incidence of ICH within 90 days of thrombolysis. Patients with WUIS were further divided into a good prognosis group (mRS score: $0-1$ points) or a poor prognosis group (mRS score $\geq 2$ points).

\section{Statistical Analyses}

Statistical analysis was performed using the Statistical Package for the Social Sciences (SPSS) version 21.0 (IBM Corp., Armonk, NY, USA). Measurement data are expressed as means \pm standard deviations. $t$-tests were used to compare data between independent groups, and paired samples $t$-tests were used to compare between paired groups. Numerical data are expressed as N (\%). Chi-squared analysis was used to compare data between groups. Influential factors were analyzed using binary logistic regression analysis. Receiver operating characteristic (ROC) curve analysis was used to evaluate the predictive value of factors related to a poor prognosis after intravenous thrombolysis in patients experiencing WUIS. A $p<0.05$ was considered statistically significant.

\section{Results}

We recruited a total of 493 patients with AIS, which included seven patients with an incomplete dataset, 12 patients who could not undergo MRI examination because of pacemakers or other internal implants, and 44 patients who underwent emergency mechanical thrombectomy. Finally, a total of 430 patients were included in our analysis. Of these, 114 cases (26.51\%) had experienced WUIS. All baseline data were compared between the WUIS and control groups. There were no significant differences between the two groups ( $p>0.05$, Table 1).

There was no significant between-group difference in NIHSS score at any of the time points or mRS score at 90 days $(p>0.05$, Table 2). The median NIHSS score was 7 points. An improvement in NIHSS score was observed in both groups at 24 hours, 3 days, and 1 week after thrombolysis $(p<0.05$, Table 2$)$. In the WUIS group, ICH occurred in five cases $(4.4 \%)$. Of these, one case $(0.9 \%)$ had symptomatic ICH, and four cases $(3.5 \%)$ had asymptomatic ICH. In the control group, ICH occurred in 14 cases (4.4\%). Of these, four cases $(1.3 \%)$ had symptomatic ICH, and 10 cases $(3.1 \%)$ had asymptomatic ICH. There were no significant differences in the proportion of patients who experienced an ICH between the two groups $(p>0.05$, Table 2).

\section{Factors Influencing Poor Prognosis After Thrombolysis in WUIS Patients}

We analyzed and compared all clinical data between the two subgroups. Univariate analysis showed that age, systolic blood pressure on admission, homocysteine level, atrial fibrillation, and NIHSS score on admission, were all significant factors that predicted poor prognosis after WUIS $(p<0.05$, Table 3). Long-term ECG monitoring was carried out for 2 weeks in patients with WUIS. Of the 114 patients monitored, 27 patients had atrial fibrillation, and 19 of these were newly diagnosed with atrial fibrillation.

Binary logistic regression analysis showed that atrial fibrillation and NIHSS score on admission were independent predictors of a poor prognosis and were independent risk factors for a poor prognosis $(p<0.05$, odds ratio [OR] $>1.0$, Table 4). ROC curve analysis showed that the areas under the curve (AUCs) for atrial fibrillation and NIHSS score on admission were 0.638 and 0.838 , respectively; sensitivity was $42.1 \%$ vs $71.1 \%$, and specificity was $85.5 \%$ vs $89.5 \%$, respectively. We then performed combined ROC analyses for these two variables using binary logistic regression, for which the combined AUC was 0.850 (Figure 1 and Table 5). 
Table I Clinical Characteristics of Patients in the Wake-Up Ischemic Stroke and Control Groups

\begin{tabular}{|c|c|c|c|c|}
\hline Variable & WUIS Group $(n=114)$ & Control Group $(n=316)$ & $\chi^{2} / \mathrm{t}$ & $P$-value \\
\hline Male (\%) & $75(65.8)$ & $205(64.9)$ & 0.031 & 0.860 \\
\hline Age (years) & $59.99 \pm 10.00$ & $60.4 \pm 10.00$ & -0.376 & 0.707 \\
\hline Systolic blood pressure $(\mathrm{mmHg})$ & $160.17 \pm 18.18$ & $155.66 \pm 27.25$ & 1.637 & 0.102 \\
\hline Diastolic blood pressure $(\mathrm{mmHg})$ & $92.82 \pm 11.12$ & $90.46 \pm 11.63$ & 1.874 & 0.062 \\
\hline Fasting blood glucose (mmol/L) & $5.88 \pm 1.66$ & $5.89 \pm 2.18$ & -0.038 & 0.969 \\
\hline Glycosylated hemoglobin (\%) & $6.10 \pm 1.38$ & $5.88 \pm 1.29$ & 1.519 & 0.130 \\
\hline Triglyceride (mmol/L) & $1.86 \pm 1.08$ & $1.69 \pm 1.01$ & 1.567 & 0.118 \\
\hline Total cholesterol (mmol/L) & $4.53 \pm 1.09$ & $4.51 \pm 1.01$ & 0.17 & 0.865 \\
\hline Low-density lipoprotein (mmol/L) & $2.99 \pm 0.99$ & $2.95 \pm 1.03$ & 0.408 & 0.683 \\
\hline Uric acid $(\mu \mathrm{mol} / \mathrm{L})$ & $298.97 \pm 97.59$ & $286.68 \pm 108.68$ & 1.063 & 0.289 \\
\hline Homocysteine $(\mu \mathrm{mol} / \mathrm{L})$ & $13.18 \pm 4.14$ & $13.82 \pm 6.15$ & -1.028 & 0.304 \\
\hline Hypertension (\%) & 77 (67.5) & $206(65.2)$ & 0.206 & 0.650 \\
\hline Diabetes (\%) & $16(14.0)$ & $60(19.0)$ & 1.412 & 0.235 \\
\hline Smoking history (\%) & $42(36.8)$ & I 44 (45.7) & 2.683 & 0.101 \\
\hline Atrial fibrillation (\%) & $27(23.7)$ & $52(16.5)$ & 2.919 & 0.088 \\
\hline Hyperlipidemia (\%) & $34(29.8)$ & $121(38.3)$ & 2.605 & 0.107 \\
\hline TOAST classification (\%) & & & 3.217 & 0.522 \\
\hline Large artery atherosclerosis & $55(48.2)$ & 161 (50.9) & & \\
\hline Small vessel occlusion & $38(33.3)$ & $113(35.8)$ & & \\
\hline Cardioembolism & $19(16.7)$ & $33(10.4)$ & & \\
\hline Other determined etiology & I (0.9) & $4(1.3)$ & & \\
\hline Undetermined etiology & $\mathrm{I}(0.9)$ & $5(1.6)$ & & \\
\hline Lesion site $(\%)$ & & & 0.008 & 0.930 \\
\hline Anterior circulation & $68(59.6)$ & $187(59.2)$ & & \\
\hline Posterior circulation & $46(40.4)$ & $129(40.8)$ & & \\
\hline NIHSS score on admission & $8.62 \pm 3.99$ & $8.38 \pm 4.07$ & 0.542 & 0.588 \\
\hline
\end{tabular}

Abbreviations: WUIS, wake-up ischemic stroke; NIHSS, National Institutes of Health Stroke Scale; TOAST, Trial of Org I0I72 in Acute Stroke Treatment.

Table 2 Comparison of Effectiveness Between the Two Groups of Patients

\begin{tabular}{|c|c|c|c|c|}
\hline Variable & WUIS Group (n = II4) & Control Group $(n=316)$ & $\chi^{2} / \mathrm{t}$ & $P$-value \\
\hline \multicolumn{5}{|l|}{ NIHSS score } \\
\hline On admission & $8.62 \pm 3.99$ & $8.38 \pm 4.07$ & 0.542 & 0.588 \\
\hline 24 hours after thrombolysis & $4.58 \pm 3.43^{*}$ & $4.20 \pm 3.88^{*}$ & 0.931 & 0.352 \\
\hline 3 days after thrombolysis & $4.23 \pm 3.47^{*}$ & $4.15 \pm 3.98 *$ & 0.188 & 0.352 \\
\hline I week after thrombolysis & $3.20 \pm 2.99 *$ & $3.06 \pm 3.35 *$ & 0.388 & 0.698 \\
\hline 90-day mRS score & $1.18 \pm 1.44$ & $0.93 \pm 1.25$ & 1.667 & 0.097 \\
\hline Intracranial hemorrhage (\%) & $5(4.4)$ & $14(4.4)$ & 0.000 & 0.984 \\
\hline
\end{tabular}

Note: *Intra-group comparison prior to thrombolysis, $p<0.05$.

Abbreviations: WUIS, wake-up ischemic stroke; NIHSS, National Institutes of Health Stroke Scale; mRS, modified Rankin Scale.

\section{Discussion}

In 2008, the ECASSIII study extended the time window for the intravenous thrombolysis of alteplase to 4.5 hours. ${ }^{9}$ After more than 10 years of exploration, an increasing body of evidence-based medicine has accumulated that supports the application of thrombolysis therapy for patients with an unknown onset time. Both the WAKE-UP study (published in 2018) and the EXTEND study (published in 2019) provided further support for the use of intravenous thrombolysis for the treatment of WUIS. ${ }^{4}$ Thrombolytic therapy is performed in patients with acute cerebral infarction for whom 9 hours have elapsed since the last normal time using either $\mathrm{CT}$ or magnetic resonance perfusion imaging, which includes patients 
Table 3 Univariate Analysis for Poor Prognosis After Intravenous Thrombolysis in Patients with Wake-Up Ischemic Stroke

\begin{tabular}{|c|c|c|c|c|}
\hline Variable & Favorable Prognosis Group $(n=76)$ & Poor Prognosis Group $(n=38)$ & $\chi^{2} / \mathbf{t}$ & $P$-value \\
\hline Male (\%) & $47(61.8)$ & $28(73.7)$ & 1.578 & 0.209 \\
\hline Age (years) & $61.33 \pm 10.3$ & $57.32 \pm 8.91$ & 2.048 & $0.043^{*}$ \\
\hline Systolic blood pressure (mmHg) & $157.62 \pm 19.34$ & $|65.26 \pm| 4.5 \mid$ & -2.363 & $0.020 *$ \\
\hline Diastolic blood pressure $(\mathrm{mmHg})$ & $92.87 \pm 12.25$ & $92.71 \pm 8.56$ & 0.071 & 0.943 \\
\hline Fasting blood glucose (mmol/L) & $5.87 \pm 1.66$ & $5.92 \pm 1.7$ & -0.159 & 0.874 \\
\hline Glycosylated hemoglobin (\%) & $6.2 \pm 1.49$ & $5.9 \pm 1.12$ & 1.084 & 0.281 \\
\hline Triglyceride (mmol/L) & $1.94 \pm 1.1$ & $1.72 \pm 1.05$ & 0.998 & 0.321 \\
\hline Total cholesterol (mmol/L) & $4.58 \pm 1.02$ & $4.43 \pm 1.23$ & 0.689 & 0.492 \\
\hline Low-density lipoprotein (mmol/L) & $3.01 \pm 0.97$ & $2.97 \pm 1.03$ & 0.204 & 0.839 \\
\hline Uric acid ( $\mu \mathrm{mol} / \mathrm{L})$ & $290.87 \pm 90.38$ & $315.18 \pm 110.1$ & -1.257 & 0.211 \\
\hline Homocysteine $(\mu \mathrm{mol} / \mathrm{L})$ & $12.58 \pm 3.78$ & $14.37 \pm 4.6$ & -2.219 & $0.029 *$ \\
\hline Hypertension (\%) & $51(67.1)$ & $26(68.4)$ & 0.020 & 0.888 \\
\hline Diabetes (\%) & II (14.5) & $5(13.2)$ & 0.036 & 0.849 \\
\hline Smoking history (\%) & $28(36.8)$ & $14(36.8)$ & 0.000 & 1.000 \\
\hline Atrial fibrillation (\%) & II (14.5) & $16(42.1)$ & 10.701 & $0.001 *$ \\
\hline Hyperlipidemia (\%) & $25(32.9)$ & $9(23.7)$ & 1.027 & 0.311 \\
\hline TOAST classification (\%) & & & 2.744 & 0.098 \\
\hline Large artery atherosclerosis & $38(50)$ & $17(44.7)$ & & \\
\hline Small-vessel occlusion & $27(35.5)$ & II (28.9) & & \\
\hline Cardioembolism & $10(13.2)$ & $9(23.7)$ & & \\
\hline Other determined etiology & I (1.3) & $0(0)$ & & \\
\hline Undetermined etiology & $0(0)$ & I (2.6) & & \\
\hline Lesion site (\%) & & & 0.018 & 0.893 \\
\hline Anterior circulation & $45(59.2)$ & $23(60.5)$ & & \\
\hline Posterior circulation & $31(40.8)$ & $15(39.5)$ & & \\
\hline NIHSS score on admission & $7 \pm 2.58$ & $11.87 \pm 4.36$ & -6.353 & $<0.001 *$ \\
\hline
\end{tabular}

Note: *Significant at $\mathrm{P} \leq 0.05$.

Abbreviations: NIHSS, National Institutes of Health Stroke Scale; TOAST, Trial of Org 10172 in Acute Stroke Treatment.

Table 4 Binary Logistic Regression Analysis for Poor Prognosis After Thrombolysis in Patients with Wake-Up Ischemic Stroke

\begin{tabular}{|c|c|c|c|c|c|c|c|}
\hline \multirow[t]{2}{*}{ Variable } & \multirow[t]{2}{*}{ B-value } & \multirow[t]{2}{*}{ SE } & \multirow[t]{2}{*}{ Wald Value } & \multirow[t]{2}{*}{$P$-value } & \multirow[t]{2}{*}{ OR } & \multicolumn{2}{|c|}{$95 \% \mathrm{Cl}$} \\
\hline & & & & & & Lower Limit & Upper Limit \\
\hline Age (year) & -0.038 & 0.027 & 2.096 & 0.148 & 0.962 & 0.914 & 1.014 \\
\hline Systolic blood pressure $(\mathrm{mmHg})$ & 0.015 & 0.015 & 1.090 & 0.297 & 1.015 & 0.987 & 1.045 \\
\hline Homocysteine $(\mu \mathrm{mol} / \mathrm{L})$ & -0.036 & 0.069 & 0.277 & 0.599 & 0.964 & 0.842 & 1.104 \\
\hline Atrial fibrillation (\%) & 1.342 & 0.584 & 5.276 & $0.022 *$ & 3.825 & 1.218 & 12.016 \\
\hline NIHSS score on admission & 0.399 & 0.090 & 19.483 & $<0.001 *$ & 1.490 & 1.248 & 1.779 \\
\hline Constant & -4.305 & 3.001 & 2.058 & 0.151 & 0.014 & & \\
\hline
\end{tabular}

Note: *Significant at $\mathrm{P} \leq 0.05$.

Abbreviations: OR, unadjusted odds ratio; $\mathrm{Cl}$, confidence interval; NIHSS, National Institutes of Health Stroke Scale; SE, standard error.

who experience WUIS. The proportion of patients in the rt-PA group with a 90-day mRS score of 0 or 1 was higher than that in the placebo group, and there was no significant difference between the two groups in terms of safety.

In our study, we used DWI-T2WI mismatch to screen patients who experienced WUIS with an unknown time of onset and provided these patients with intravenous thrombolytic therapy. We found that effectiveness did not differ between patients with non-WUIS within $4.5 \mathrm{~h}$ and WUIS patients. In the first few hours following the onset of AIS, an MRI of the brain reveals a characteristic dynamic process. The initial destruction of cellular energy metabolism leads to cytotoxic edema, which can be seen within a few minutes owing to a decrease in the apparent diffusion coefficient on DWI. In the following hours, cell liquefaction and necrosis lead to an increase in tissue osmotic pressure, as shown in T2WI. Therefore, a negative T2WI signal indicates a delay in damage to the blood-brain barrier and therefore, a delay in 


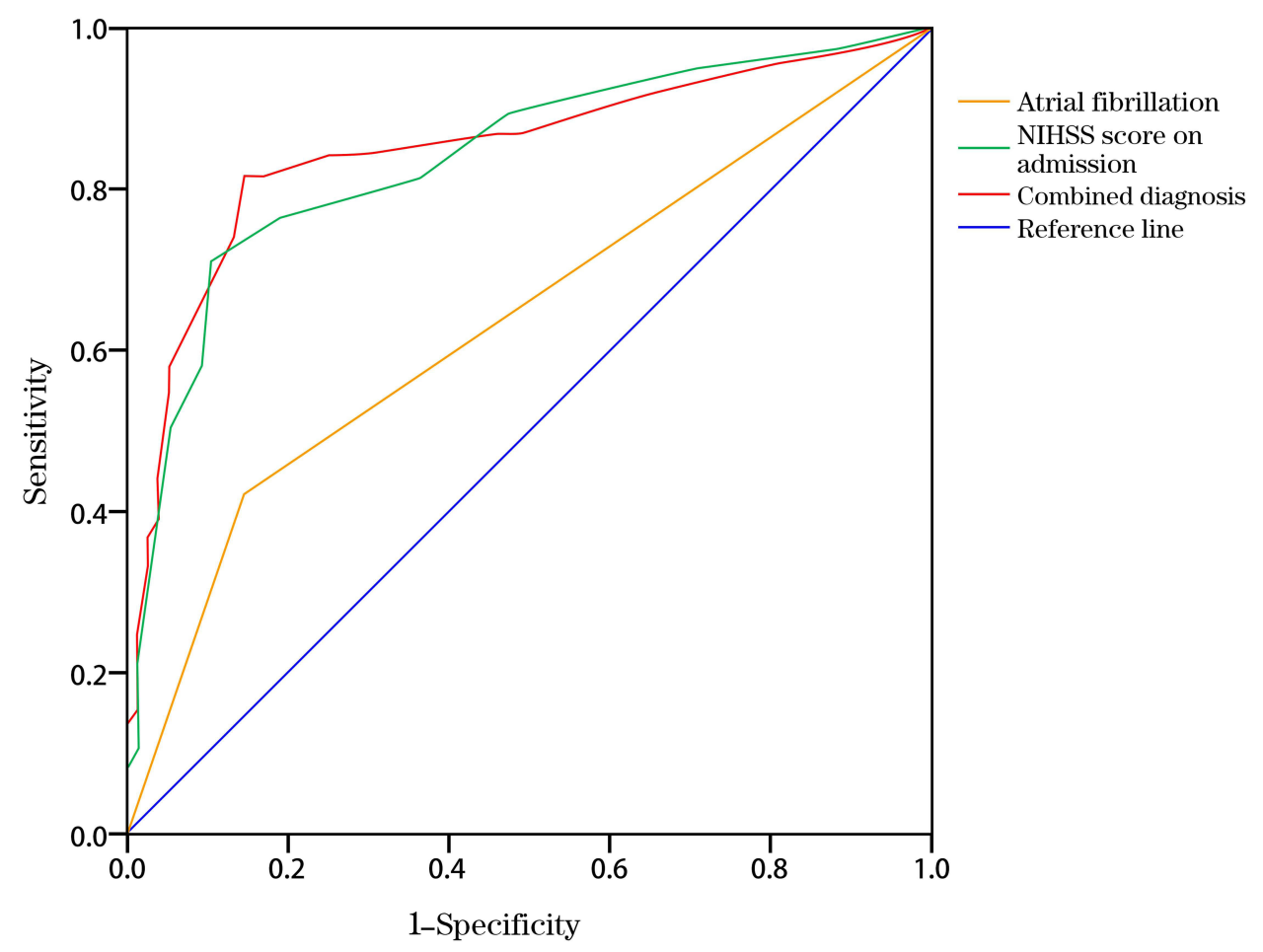

Figure I Receiver operating characteristic curves.

vasogenic edema. This suggests that the stroke occurred during approximately the previous $4.5 \mathrm{~h}$, which is the accepted interval for treatment with thrombolysis. Our previous research, which was carried out in a single center, showed that the acquisition of T2WI data $(50 \mathrm{~s})$ is approximately $100 \mathrm{~s}$ faster than acquiring FLAIR images. ${ }^{10}$ Although the $100 \mathrm{~s}$ saved by acquiring T2WI may seem minimal, the sequence is simple and easy to implement. Indeed, the slogan of World Stroke Day 2021 was "Every second counts, heal the brain." Recognizing a stroke even $1 \mathrm{~s}$ earlier provides an additional opportunity to preserve brain function and allow for better recovery. Consequently, every second is crucial if we are to restore the ischemic penumbra. We cannot predict and control the pre-hospital delay; thus, we must develop methods to reduce the delay in hospital and improve factors that are within our control. Such factors include time-consuming MRI examination, which is necessary and beneficial for WUIS patients because of the unknown onset time. Therefore, we consider DWI-T2WI mismatch a reliable method for the rapid evaluation of the penumbra that is as accurate as the outcomes reported in the WAKE-UP and MR WITNESS trials.

With regards to the risk factors and etiological classifications of WUIS, numerous authors have reported that there are no significant differences between patients who experience WUIS and those who have a non-WUIS., ${ }^{31,12}$ We compared the two patient groups with regards to sex, age, smoking, previous and newly diagnosed diseases (eg, hypertension,

Table 5 Binary Logistic Regression Analysis for the Combined Receiver Operating Curve Diagnosis

\begin{tabular}{|l|c|c|c|c|c|c|c|}
\hline Variable & \multirow{2}{*}{ AUC } & \multirow{2}{*}{ SE } & \multirow{2}{*}{ P-value } & \multicolumn{2}{|c|}{$\mathbf{9 5 \%}$ Cl } & \multirow{2}{*}{ Sensitivity } & \multirow{2}{*}{ Specificity } \\
\cline { 5 - 7 } & & & & $\begin{array}{c}\text { Lower } \\
\text { Limit }\end{array}$ & $\begin{array}{c}\text { Upper } \\
\text { Limit }\end{array}$ & & \\
\hline Atrial fibrillation & 0.638 & 0.058 & $0.016^{*}$ & 0.525 & 0.751 & $42.10 \%$ & $85.50 \%$ \\
NIHSS score on admission & 0.838 & 0.043 & $<0.001 *$ & 0.755 & 0.922 & $71.10 \%$ & $89.50 \%$ \\
Combined diagnosis & 0.850 & 0.043 & $<0.001 *$ & 0.765 & 0.935 & $81.60 \%$ & $85.50 \%$ \\
\hline
\end{tabular}

Notes: ${ }^{\#}$ Combined atrial fibrillation and NIHSS score on admission; *Significant at $\mathrm{P} \leq 0.05$.

Abbreviations: AUC, area under the curve; Cl, confidence interval; NIHSS, National Institutes of Health Stroke Scale; SE, standard error. 
hyperlipidemia, diabetes, and atrial fibrillation), systolic/diastolic blood pressure on admission, TOAST classification, NIHSS score, lesion site (ie, anterior or posterior circulation), and common laboratory biochemical indices, of which none were significantly different. This suggests that these two groups share risk factors and pathogenic pathways.

Very few studies have investigated the factors that contribute to a poor prognosis in patients with WUIS. Most researchers believe that the factors that lead to a poor prognosis in WUIS patients are complex and diverse and may arise from the combined action of multiple factors. ${ }^{13}$ In our study, we found that baseline NIHSS score and atrial fibrillation were independent predictive factors of a poor prognosis after intravenous thrombolytic therapy in patients with WUIS. NIHSS score is a quantitative parameter that directly reflects the degree of neurological deficit. A higher score indicates that the lesion size is larger or that the infarction site is located in a functionally important brain region, thus leading to serious neurological deficits and a poor prognosis. Therefore, the NIHSS score has high sensitivity and specificity for evaluating prognosis. ${ }^{14}$

Because the symptoms of WUIS are usually detected in the morning, the condition is currently considered to be related to the rhythmic fluctuation of cardiovascular autonomic nervous system function. In our study, we observed that the rate of new atrial fibrillation was $16.7 \%$ (19/114) in patients with WUIS after 2 weeks of ECG monitoring, which was 2.4-times higher than that after $24 \mathrm{~h}$ of ECG monitoring. Although the occurrence of atrial fibrillation showed a bimodal rhythm at midnight and 1:00 PM, the most frequent termination and conversion to sinus rhythm tended to occur at approximately 6:00 AM. This may explain the association between atrial fibrillation and WUIS. ${ }^{15}$ Atrial fibrillation is one of the most common arrhythmias detected in the clinic and significantly increases the risk of cerebral embolism. Furthermore, the emboli formed by atrial fibrillation are usually large and generally unstable In addition to causing severe hypoperfusion, atrial fibrillation leads to embolus shedding or disintegration, vascular recanalization, and blood flow reperfusion, which induces reperfusion injury and even secondary bleeding; ultimately, resulting in a poor clinical prognosis.

In our study, six patients $(63.6 \%)$ in the good prognosis group with atrial fibrillation received anticoagulants: four patients were on warfarin, and two were on rivaroxaban. The patients were continuing to take anticoagulants regularly at the 90-day follow-up examination. Eight patients $(50 \%)$ in the poor prognosis group with AF received anticoagulants; five were on warfarin and three on rivaroxaban. At the 90-day follow-up examination, two patients had discontinued rivaroxaban because of its high cost, and one had discontinued warfarin because of an unwillingness to frequently monitor international normalized ratio. At present, oral anticoagulants remain the first-line treatment for the prevention of atrial fibrillation-related stroke. However, in China, anticoagulant therapy for patients with atrial fibrillation combined with ischemic stroke continues to be met with numerous challenges, such as a low anticoagulant rate, a high withdrawal rate, the limited use of non-vitamin $\mathrm{K}$ antagonist oral anticoagulants, and poor patient compliance. ${ }^{16} \mathrm{In}$ addition, because of the irregular frequency of the short-term and asymptomatic paroxysms of AF, it is extremely difficult to rely on traditional detection methods for clinical diagnoses, such as conventional ECG and 24-hour dynamic ECG; this often leads to missed diagnoses and delayed treatment. Therefore, it is important that we improve and standardize anticoagulation treatments for atrial fibrillation. High-level hospitals could adopt new atrial fibrillation screening and monitoring techniques (eg, portable ECG monitoring patches and smartwatches with ECG monitoring functions) to improve the detection rate of atrial fibrillation. ${ }^{17}$ These measures will significantly benefit our ability to prevent and manage atrial fibrillation.

The ROC curve analysis showed that the combination of atrial fibrillation and NIHSS score was a better predictor of a poor prognosis after intravenous thrombolysis than when they were considered independently (AUC: $0.850>0.838>$ $0.638)$. Furthermore, the combination of these factors retained a high level of sensitivity $(81.6 \%)$ and specificity $(85.5 \%)$. The AUC for the combined diagnosis was only minimally different from that for the NIHSS score $(0.850>0.838)$, which indicated that the diagnostic efficacy of these two indicators was similar. We put forward two reasons for this result. First, the NIHSS score directly reflects the severity of neurological deficits and is more closely related to disease prognosis, which may have a greater influence on the combined diagnosis. Second, the similarity may be related to the bias introduced by the small number of patients with AF. However, in terms of sensitivity, the value of the combined diagnosis was significantly better than that of the NIHSS score $(81.6 \%>71.1 \%)$, and it could reduce the missed diagnosis rate of a poor prognosis. It is well established that stroke patients with a poor prognosis place heavy burdens on 
their families and society. Therefore, we need to minimize missed diagnoses, improve early detection of strokes, and actively treat the disease in the early stages to prevent poor prognoses. Thus, we consider that the predictive value of a combined diagnosis is greater than that of single indicator. When physicians encounter WUIS patients with AF and a high baseline NIHSS score, they must consider the possibility of a poor prognosis and be highly vigilant during treatment.

There are some limitations to our study that must be considered. First, this was a retrospective study, and our sample size of patients with WUIS was not sufficient to accurately identify all characteristics. In addition, several studies have reported that cerebral microbleeds are an independent risk factor for the transformation of ICH after stroke thrombolysis; moreover, they are associated with a significantly increased risk of a poor functional outcome. ${ }^{18,19}$ Regrettably, our study did not include cerebral microbleeds; this factor should be considered in future research. In the future, it will be important to consider prospective, multi-center clinical studies, with large sample sizes to provide more evidence-based data. This would allow a more comprehensive understanding of WUIS.

\section{Conclusion}

The DWI-T2WI sequence is an effective method to guide intravenous thrombolytic therapy for WUIS. Baseline NIHSS score and atrial fibrillation were identified as independent predictors of poor prognosis following thrombolysis in WUIS patients. The combination of the two variables had a higher predictive value for a poor prognosis after thrombolysis in patients with WUIS. Early screening using adequate imaging, stroke severity assessment, and long-range ECG monitoring will help guide clinical treatment and predict prognosis in patients with WUIS.

\section{Ethics Statement}

This study was approved by the Medical Ethics Committee of Shanghai Eighth People's Hospital and Pudong New Area People's Hospital. This study was conducted in accordance with the Declaration of Helsinki. This was a retrospective clinical study that only collected the clinical data of patients. Therefore, it did not interfere with patients' treatment plans, involve human body research, or risk the physiology and psychology of patients. Another reason is because of the long duration of our study, regrettably, some patients have died. However, we guarantee that the research data are authentic and reliable and that we will always protect the privacy of our patients. Therefore, the ethics committee exempted the requirement for obtaining informed consent from patients.

\section{Funding}

This research was supported by the National Natural Science Foundation of China (81870920, 81801262), the Key Discipline Group Construction Project of Pudong Health Bureau of Shanghai (PWZxq2017-02), and the Featured Clinical Discipline Project of Shanghai Pudong (PWYst2018-01).

\section{Disclosure}

The authors report no conflicts of interest in this work.

\section{References}

1. Thomalla G, Boutitie F, Fiebach JB, et al. Stroke with unknown time of symptom onset: baseline clinical and magnetic resonance imaging data of the first thousand patients in WAKE-UP (efficacy and safety of MRI-based thrombolysis in wake-up stroke: a randomized, double-blind, placebocontrolled trial). Stroke. 2017;48(3):770-773.

2. Rehani B, Ammanuel SG, Zhang Y, et al. A new era of extended time window acute stroke interventions guided by imaging. Neurohospitalist. 2020;10(1):29-37. doi:10.1177/1941874419870701

3. Thomalla G, Simonsen CZ, Boutitie F, et al. MRI-guided thrombolysis for stroke with unknown time of onset. N Engl J Med. 2018;379(7):611-622. doi:10.1056/NEJMoa1804355

4. Ma H, Campbell BCV, Parsons MW, et al. Thrombolysis guided by perfusion imaging up to 9 hours after onset of stroke. $N$ Engl J Med. 2019;380 (19):1795-1803. doi:10.1056/NEJMoa1813046

5. Nagai K, Aoki J, Sakamoto Y, Kimura K. About $30 \%$ of wake-up stroke patients may be candidates for the tPA therapy using negative-FLAIR as a "tissue clock". J Neurol Sci. 2017;382:101-104. doi:10.1016/j.jns.2017.09.042

6. Campbell BCV, Ma H, Ringleb PA, et al. Extending thrombolysis to $4.5-9 \mathrm{~h}$ and wake-up stroke using perfusion imaging: a systematic review and meta-analysis of individual patient data. Lancet. 2019;394(10193):139-147. doi:10.1016/S0140-6736(19)31053-0 
7. Darehed D, Blom M, Glader EL, Niklasson J, Norrving B, Eriksson M. In-hospital delays in stroke thrombolysis: every minute counts. Stroke. 2020;51(8):2536-2539. doi:10.1161/STROKEAHA.120.029468

8. Meretoja A, Keshtkaran M, Saver JL, et al. Stroke thrombolysis: save a minute, save a day. Stroke. 2014;45(4):1053-1058. doi:10.1161/ STROKEAHA.113.002910

9. Hacke W, Kaste M, Bluhmki E, et al. Thrombolysis with alteplase 3 to 4.5 hours after acute ischemic stroke. $N$ Engl J Med. 2008;359(13):13171329. doi:10.1056/NEJMoa0804656

10. Bai QK, Zhao ZG, Lu LJ, et al. Treating ischaemic stroke with intravenous tPA beyond 4.5 hours under the guidance of a MRI DWI/T2WI mismatch was safe and effective. Stroke Vasc Neurol. 2019;4(1):8-13. doi:10.1136/svn-2018-000186

11. Mackey J, Kleindorfer D, Sucharew H, et al. Population-based study of wake-up strokes. Neurol. 2011;76(19):1662-1667. doi:10.1212/ WNL.0b013e318219fb30

12. Wouters A, Lemmens R, Dupont P, Thijs V. Wake-up stroke and stroke of unknown onset: a critical review. Front Neurol. $2014 ; 5(5): 153$. doi:10.3389/fneur.2014.00153

13. Jovin TG. MRI-guided intravenous alteplase for stroke-still stuck in time. $N$ Engl J Med. 2018;379(7):682-683. doi:10.1056/NEJMe1805796

14. Mokin M, Masud MW, Dumont TM, et al. Outcomes in patients with acute ischemic stroke from proximal: intracranial vessel occlusion and NIHSS score below 8. J Neurointerv Surg. 2014;6(6):413-417. doi:10.1136/neurintsurg-2013-010720

15. Corino VD, Platonov PG, Enger S, Tveit A, Ulimoen SR. Circadian variation of variability and irregularity of heart rate in patients with permanent atrial fibrillation: relation to symptoms and rate control drugs. Am J Physiol Heart Circ Physiol. 2015;309(12):H2152-2157. doi:10.1152/ ajpheart.00300.2015

16. Guo J, Guan T, Fan S, Chao B, Wang L, Liu Y. Underuse of oral anticoagulants in patients with ischemic stroke and atrial fibrillation in China. Am J Cardiol. 2018;122(12):2055-2061. doi:10.1016/j.amjcard.2018.08.057

17. Steinhubl SR, Waalen J, Edwards AM, et al. Effect of a home-based wearable continuous ECG monitoring patch on detection of undiagnosed atrial fibrillation: the mSToPS randomized clinical trial. JAMA. 2018;320(2):146-155. doi:10.1001/jama.2018.8102

18. Charidimou A, Turc G, Oppenheim C, et al. Microbleeds, cerebral hemorrhage, and functional outcome after stroke thrombolysis. Stroke. 2017;48 (8):2084-2090. doi:10.1161/STROKEAHA.116.012992

19. Tsivgoulis G, Zand R, Katsanos AH, et al. Risk of symptomatic intracerebral hemorrhage after intravenous thrombolysis in patients with acute ischemic stroke and high cerebral microbleed burden: a meta-analysis. JAMA Neurol. 2016;73(6):675-683. doi:10.1001/jamaneurol.2016.0292

Neuropsychiatric Disease and Treatment

Dovepress

\section{Publish your work in this journal}

Neuropsychiatric Disease and Treatment is an international, peer-reviewed journal of clinical therapeutics and pharmacology focusing on concise rapid reporting of clinical or pre-clinical studies on a range of neuropsychiatric and neurological disorders. This journal is indexed on PubMed Central, the 'PsycINFO' database and CAS, and is the official journal of The International Neuropsychiatric Association (INA). The manuscript management system is completely online and includes a very quick and fair peer-review system, which is all easy to use. Visit http://www.dovepress.com/testimonials.php to read real quotes from published authors.

Submit your manuscript here: https://www.dovepress.com/neuropsychiatric-disease-and-treatment-journal 